\title{
Senescence of fibroblastic reticular cells in draining lymph nodes: immunoregulation following transplantation
}

\author{
Zhaoli Sun and James Burdick \\ Department of Surgery, Johns Hopkins University School of Medicine, Baltimore, Maryland, USA.
}

\begin{abstract}
The lymph node (LN) is an intriguing site not only for inducing protective effector immunity but also for inducing tolerance against peripherally encountered antigens such as tissue-specific self-antigens that are regionally drained and through draining lymph nodes (DLNs). The dual functions of DLNs in immunity are attributable at least in part to fibroblastic reticular cells (FRCs), which are a major population of the nonhematopoietic compartment in the LN. In this issue of the JCI, Li, Zhao, and colleagues investigated DLNs in the transplantation setting. The authors demonstrated that, following skin transplantation, the donor mast cell-mediated senescence in FRCs was associated with collagen 1 deposition in DLNs. Systemic administration to mice of FRCs that were expanded ex vivo decreased DLN fibrosis and strengthened the effect of anti-CD40L in prolonging heart allograft survival. These data implicate the DLN as a target for immunomodulatory therapy of transplant rejection.
\end{abstract}

Lymph nodes provide an ideal microenvironment for communication

The lymph node (LN), a secondary lymphoid organ, plays an integral role in the immune functions of the body. In addition to T cells, B cells, DCs, macrophages, and plasma cells, the LN contains specialized stromal cells including blood endothelial cells (BECs), lymphatic endothelial cells (LECs), follicular DCs (FDCs), marginal reticular cells (MRCs), integrin $\alpha 7^{+}$pericytes (IAPs), and fibroblastic reticular cells (FRCs) $(1,2)$. LNs provide an ideal microenvironment for communication between immune cells and serve as a central site for recruiting naive $\mathrm{T}$ cells from the blood, promoting naive $\mathrm{T}$ cell survival, providing an environment for $\mathrm{T}$ cell differentiation for effector roles or tolerance, and influencing the homing properties of memory $\mathrm{T}$ cells (3).

FRCs are a major population of the nonhematopoietic compartment in LNs and comprise $20 \%-50 \%$ of stromal cells (2). FRCs organize the LN microarchitecture and produce extracellular matrix (ECM) components to create the conduit network, which rapidly transports soluble antigens and signaling molecules deep into the LN parenchyma and directs $\mathrm{T}$ and $\mathrm{B}$ cell trafficking using chemokine secretion. FRCs are constantly in contact with naive $\mathrm{T}$ cells and DCs while these cells are migrating along the network and scanning for antigen-specific affinity (1). FRCs not only support the structure for immune cell interaction but also exhibit several fascinating immuno-

Related Article: p. 4182

Conflict of interest: ZS holds equity in, consults for, and is an advisor to MedRegan LLC. Additionally, ZS is an inventor of technology (patent no. US 10,420,751 B2; Methods of Treating Inflammatory Bowel Disease) and participates in research funded by MedRegen LLC, which intends to further develop the technology.

Copyright: @ 2020, American Society for Clinical Investigation.

Reference information: / Clin Invest. 2020;130(8):3965-3967. https://doi.org/10.1172/JCI139153.

regulatory properties (4-6). FRCs are concentrated in the paracortical region ( $\mathrm{T}$ cell zone) of the LN and are endowed with several functions that regulate the activity of $\mathrm{T}$ lymphocytes. When a naive $\mathrm{T}$ cell becomes activated in the $\mathrm{LN}$ by a DC under regulation by FRCs, the $\mathrm{T}$ cell will either mount an effector response or will become tolerant to avoid autoimmunity $(7,8)$. The intimate contact with immune cells puts FRCs at the front line of the immune response, where they fundamentally regulate adaptive immunity. Emerging data suggest that normal functioning of the FRC network is essential to immunological health. Loss of FRCs results in $60 \%-70 \%$ depletion of $\mathrm{T}$ cells, B cells, DCs, and macrophages and impairs systemic immune responses to virus infection (9). Viral pathology drives FRC-mediated LN fibrosis resulting in immunodeficiency (10). There are few studies of FRCs in the transplant setting.

In this issue of the JCI, Li, Zhao, and colleagues (11) demonstrated that the donor mast cell-mediated senescence in FRCs occurs through an interaction between LIGHT (also called TNF superfamily member 14 [TNFSF14]) and HVEM (the herpes virus entry mediator, a member of the TNF receptor superfamily). LIGHT-HVEM interaction was associated with collagen 1 deposition in draining LNs (DLNs) following skin transplantation. Senescence in FRCs resulted in massive production of collagen 1 and a proinflammatory milieu within the DLN. When Li , Zhao, and colleagues systemically administered FRCs that were expanded ex vivo to mice, DLN fibrosis decreased and anti-CD40L more effectively prolonged heart allograft survival. This finding provides insight into DLN-mediated alloimmunity and is an important step toward a better understanding of FRC-mediated immunomodulation. Several issues are worth highlighting. 


\section{Immunoregulatory function of FRCs in LNs}

Despite supporting naive $\mathrm{T}$ cell migration and proliferation in the LN, FRCs also play an essential role in regulating $\mathrm{T}$ cell-mediated immune responses. FRCs contribute to $\mathrm{T}$ cell tolerance in 2 major ways. First, FRCs serve as antigen-presenting cells and can directly present tissue-specific self-antigens to $\mathrm{CD}^{+} \mathrm{T}$ cells for tolerance induction (12). Presentation of peripheral tissue-restricted antigens by FRCs prevents autoimmunity in animal models by directly deleting autoreactive $\mathrm{T}$ cell clones (4). Direct infection of FRCs was associated with an inability to clear the virus and the acquisition of an exhausted phenotype in responding $\mathrm{CD}^{+} \mathrm{T}$ cells (13). Second, FRCs support Tregs: OVA-expressing FRCs were found to promote OVA-specific Tregs in a K14-OVA mouse model (14). Li, Zhao, and colleagues (11) found that addition of healthy FRCs in DLNs increased Foxp3 ${ }^{+}$ Tregs, indicating the importance of FRCs in regulating alloimmunity.

\section{Senescence impairs immunoregulatory function of FRCs}

Senescence is a state of permanent cell growth arrest without accompanying cell death in the response to sustained stress over longer periods of time. Senescence can be induced by unrepaired DNA damage or other cellular stresses. Production of inflammatory cytokines and chemokines is essential for the induction and maintenance of senescence. Maarouf et al. (15) reported that repetitive renal ischemia-reperfusion injury was associated with senescence of FRCs, fibrosis of the kidney LN, and renal scarring, which were ameliorated by administration of healthy FRCs. Li, Zhao, and colleagues (11) found that donor mast cells from allografts migrated into DLNs 2 hours after transplantation and that these mast cells induced senescence in FRCs, probably through a proinflammatory milieu and LIGHT-HVEM interaction. Senescence in FRCs increased the expression of ECM genes and IL-6, the key cytokine linked to the senescenceassociated secretory phenotype (SASP). The authors observed that persistence of proinflammatory cytokines due to senescence in FRCs resulted in collagen 1

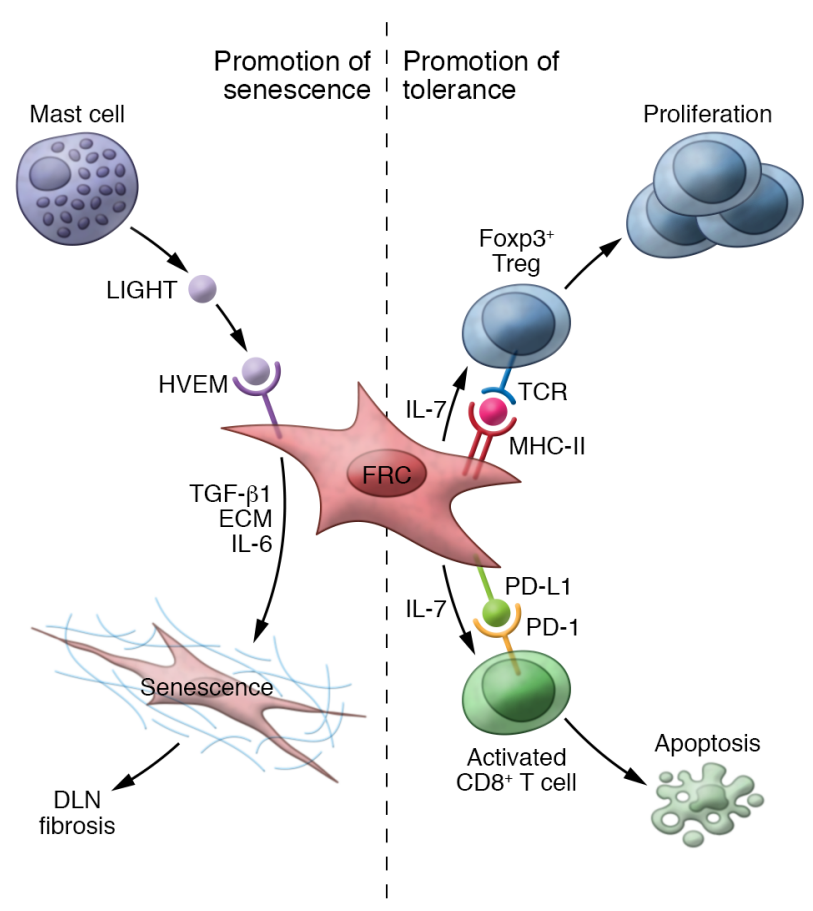

Figure 1. Model for donor mast cell-mediated senescence of FRCs. Li, Zhao, and colleagues showed that following transplantation, collagen 1 was deposited in DLNs and fibrosis ensued. Donor mast cells induced senescence of FRCs through LICHT-HVEM interaction (11). Healthy FRCs deleted activated CD8 ${ }^{+} T$ cells and supported Foxp3 ${ }^{+}$Tregs to promote tolerance. Systemic administration of FRCs increased Foxp $3^{+}$Tregs and promoted tolerance (11). TCR, T cell receptor.

deposition and fibrosis in the DLN. DLN fibrosis impaired the microarchitecture and immunoregulatory function of FRCs, whereas administration of healthy FRCs reduced the proportion of senescent FRCs, increased Foxp3 ${ }^{+}$Tregs in DLNs, and prolonged cardiac allograft survival following transplantation. Thus, senescence in FRCs impairs their immunoregulatory function, whereas administration of healthy FRCs may restore this FRC function in the DLN (Figure 1).

\section{Allogeneic mast cells, senescence in FRCs, and DLN fibrosis}

Mast cells are present in connective tissues throughout the body and serve multiple functions including effecting or regulating allergic responses, innate and adaptive immunity, autoimmunity, and inflammation. Mast cells serve as mediators of fibrosis and effector cell recruitment through chemokine production in dermal chronic graft-versus-host disease following bone marrow transplantation (16). Li, Zhao, and colleagues (11) demonstrated that ischemia and oxidative stress in mice increased the expression of mast cell proteases (mMCP-2, $\mathrm{mMCP}-4$, and $\mathrm{mMCP}-$ 6), VEGF, FGF-2, and IL-6. VEGF could support lymphatic expansion, FGF-2 could stimulate FRCs, and IL-6 could create a proinflammatory milieu in the DLN and promote senescence, respectively. Cell culture studies further demonstrated that supernatant collected from a mast cell line treated with hydrogen peroxide increased the expression of collagen 1 , TGF- $\beta 1$, and SMAD2 by FRCs and promoted lymphangiogenesis in vitro using SVEC4-10. However, collagen 1 deposition in the DLNs of the syngeneic transplant recipients was considerably lower than in those of the allogeneic transplant recipients (11). It seems that allogeneic mast cells are more potent for enhancing senescence in FRCs and DLN fibrosis, an observation that warrants further investigation.

\section{Targeting donor mast cells and FRCs in the DLN}

The report from Li, Zhao, and colleagues (11) suggested a few therapeutic strategies to treat transplant rejection. Their data underscore the role of LNs as a second site of $\mathrm{T}$ 
cell education after the thymus, which only eliminates harmful autoreactive cells. The LNs undertake the subtle job of directing the $\mathrm{T}$ cells against harmful antigens or alloantigens, but also retain a role of restraining overresponsiveness. This directing/ restraining process might be more important for allograft rejection than sensitization by circulating host cells within allograft tissue. Moreover, as these data suggest, LN biology may be a better place to seek options for therapeutic immunosuppression.

Deletion of mast cells from a donor graft or blocking mast cell migration may prevent collagen 1 deposition in DLNs. Preventing senescence in FRCs by blocking LIGHTHVEM interaction may optimally suppress the immune system. Antifibrotic medications may also help to prevent DLN fibrosis. Regardless, administration of healthy FRCs and reducing the proportion of senescent FRCs in the DLN seem a practical way to maintain immunoregulatory functions of FRCs following transplantation.

Address correspondence to: Zhaoli Sun, Department of Surgery, Johns Hopkins
University School of Medicine, 720 Rutland Avenue, Ross 771, Baltimore, Maryland 21205, USA. Email: zsun2@jhmi.edu.

1. Bajénoff M, et al. Stromal cell networks regulate lymphocyte entry, migration, and territoriality in lymph nodes. Immunity. 2006;25(6):989-1001.

2. Fletcher AL, et al. Reproducible isolation of lymph node stromal cells reveals site-dependent differences in fibroblastic reticular cells. Front Immunol. 2011;2:35

3. Fletcher AL, Acton SE, Knoblich K. Lymph node fibroblastic reticular cells in health and disease. Nat Rev Immunol. 2015;15(6):350-361.

4. Lee JW, et al. Peripheral antigen display by lymph node stroma promotes $\mathrm{T}$ cell tolerance to intestinal self. Nat Immunol. 2007;8(2):181-190.

5. Yip L, et al. Deaf1 isoforms control the expression of genes encoding peripheral tissue antigens in the pancreatic lymph nodes during type 1 diabetes. Nat Immunol. 2009;10(9):1026-1033.

6. Fletcher AL, et al. Lymph node fibroblastic reticular cells directly present peripheral tissue antigen under steady-state and inflammatory conditions. J Exp Med. 2010;207(4):689-697.

7. Lukacs-Kornek V, et al. Regulated release of nitric oxide by nonhematopoietic stroma controls expansion of the activated $\mathrm{T}$ cell pool in lymph nodes. Nat Immunol. 2011;12(11):1096-1104.

8. Khan O, Headley M, Gerard A, Wei W, Liu L,
Krummel MF. Regulation of T cell priming by lymphoid stroma. PLoS ONE. 2011;6(11):e26138.

9. Chai Q, et al. Maturation of lymph node fibroblastic reticular cells from myofibroblastic precursors is critical for antiviral immunity. Immunity. 2013;38(5):1013-1024.

10. Estes JD. Pathobiology of HIV/SIV-associated changes in secondary lymphoid tissues. Immunol Rev. 2013;254(1):65-77.

11. Li, et al. Lymph node fibroblastic reticular cells deposit fibrosis-associated collagen following organ transplantation. JClin Invest. 2020;130(8):4182-4194.

12. Fletcher AL, et al. Lymph node fibroblastic reticular cells directly present peripheral tissue antigen under steady-state and inflammatory conditions. J Exp Med. 2010;207(4):689-697.

13. Mueller SN, et al. Viral targeting of fibroblastic reticular cells contributes to immunosuppression and persistence during chronic infection. Proc Natl Acad Sci USA. 2007;104(39):15430-15435.

14. Baptista AP, et al. Lymph node stromal cells constrain immunity via MHC class II self-antigen presentation. Elife. 2014;3:e04433.

15. Maarouf $\mathrm{OH}$, et al. Repetitive ischemic injuries to the kidneys result in lymph node fibrosis and impaired healing. JCI Insight. 2018;3(13):120546

16. Strattan E, et al. Mast Cells Are Mediators of Fibrosis and Effector Cell Recruitment in Dermal Chronic Graft-vs.-Host Disease. Front Immunol. 2019;10:2470. 